\title{
HORUS, an HPAD X-ray detector simulation program
}

\author{
G. Potdevin, ${ }^{1}$ U. Trunk and H. Graafsma \\ DESY, \\ Notkestr. 85, 22607 Hamburg, Germany \\ E-mail: guillaume.potdevin@desy.de
}

ABSTRACT: The development of modern X-ray detectors is a complex process, and it is often difficult to evaluate the effect of each single technological choice on the overall performance of the detector. HORUS (HPAD Output Response fUnction Simulator) is a 2D detector simulation program simulating all the physical and electronic processes impacting the detective quantum efficiency of the detector.

The program has a modular and pluggable structure, making it easy to deactivate or modify each of the processes implemented, and thus to evaluate the impact of each of them on the detector performances.

In addition, HORUS is a very helpful tool to evaluate the influence of the detector on the scientific output. This is especially interesting in the case of experiments which were never carried out before, like those which will be done at the future hard X-ray XFEL facilities coming online in the coming months and years.

Last, HORUS is a great educational tool for anybody who wants to learn about the behaviour and working mechanism of a detector.

KEYWORDS: Detector modelling and simulations II (electric fields, charge transport, multiplication and induction, pulse formation, electron emission, etc); Instrumentation for FEL; Detector modelling and simulations I (interaction of radiation with matter, interaction of photons with matter, interaction of hadrons with matter, etc)

\footnotetext{
${ }^{1}$ Corresponding author.
} 


\section{Contents}

1 Introduction 1

2 Description of the program 2

2.1 Overall structure 4

2.2 Geometry 5

2.3 Sensor simulation 6

2.4 Readout electronics simulation 9

3 Example of uses of HORUS: Study of the AGIPD 10

3.1 Description of the AGIPD and its simulation 10

$\begin{array}{lll}3.1 .1 & \text { The AGIPD } & 10\end{array}$

3.1.2 AGIPD specific simulation models in HORUS 11

$\begin{array}{lll}3.2 & \text { Study of the AGIPD statistical response } & 13\end{array}$

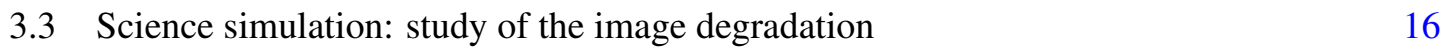

$\begin{array}{lll}3.4 & \text { Educational aspect of HORUS } & 18\end{array}$

$\begin{array}{lll}4 & \text { Summary } & 19\end{array}$

\section{Introduction}

X-ray Free Electron Lasers (FELs) constitute the next generation of X-ray photon sources, surpassing the existing third generation synchrotron sources by several orders of magnitude in many of the performance parameters. Their bunch lengths will be less than 100 femto-seconds, the light almost fully coherent, and the pulse intensity as high as $10^{12}$ photons. This leads to an increase in peak brilliance of 9 orders of magnitude as compared to the synchrotron sources. It is therefore evident that FELs will open the road to a whole range of new experiments, such as ultra fast coherent diffraction imaging, high energy density matter experiments, or femto-second time resolved experiments [1]. Various FELs are under commissioning [2] or construction [1,3] throughout the world. Among these the European XFEL based in Hamburg (Germany) will feature the highest luminosity, thanks to the super-conducting accelerator technology used. The photon pulses will be produced at a repetition rate of $5 \mathrm{MHz}$, in trains of 3000 bunches, and with a train repetition rate of $10 \mathrm{~Hz}$ (see figure 1) [1, 4], thus giving 30000 bunches per second. This offers exciting possibilities, but at the same time poses additional challenges for the experiments in general and for the large area X-ray detectors, needed for many of the foreseen experiments, in particular.

The development of these 2-dimensional X-ray detectors is extremely challenging with requirements beyond anything that is available today. In addition, many of the experiments will be completely new, and at this moment still not fully defined. This means that the development of the 


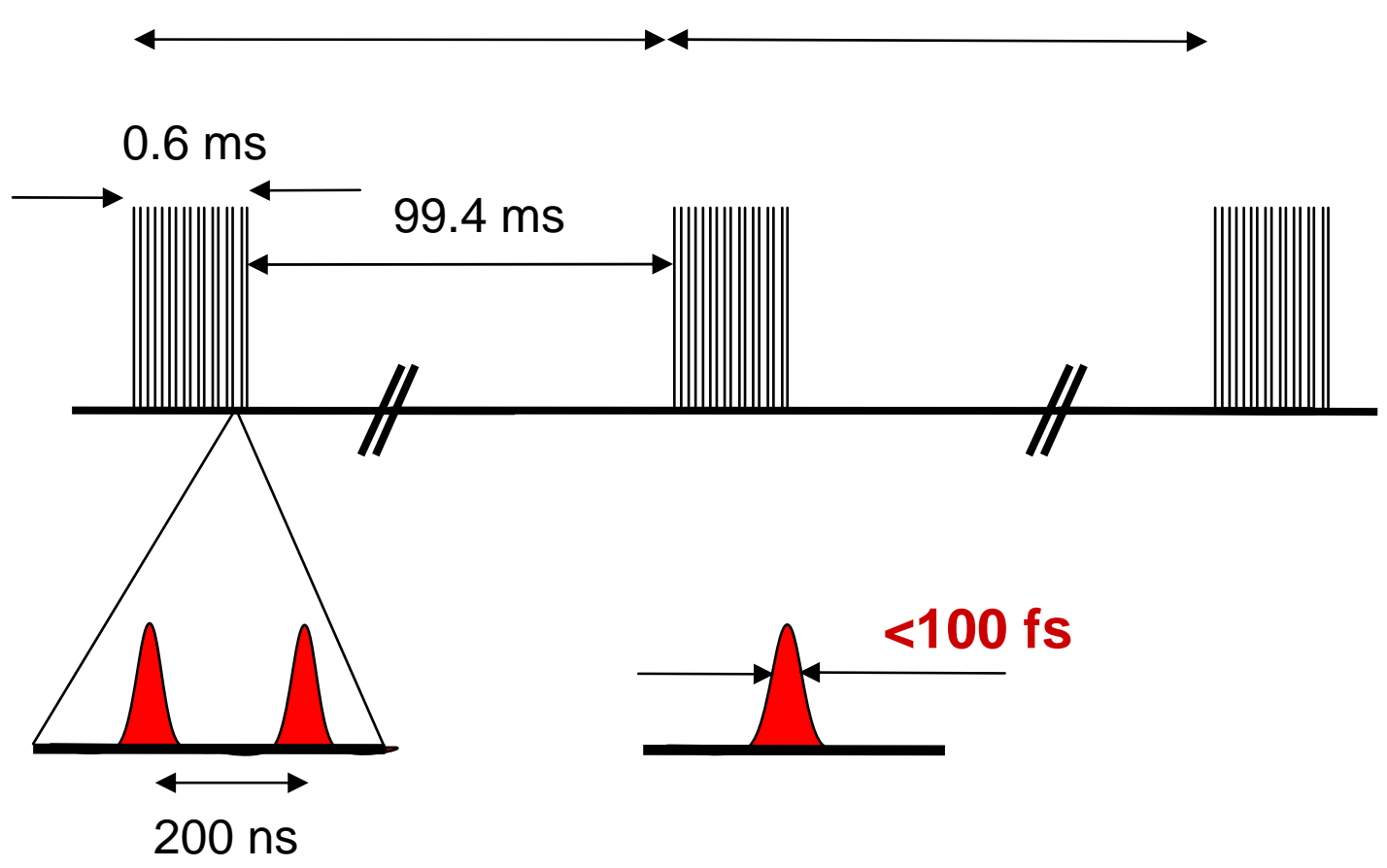

Figure 1. The European XFEL photon bunches time structure.

detectors will have to go hand-in-hand with the development of the experiments. In order to steer this development process, and to ensure a good match of the detector performances with the scientific needs, it is necessary to perform complete simulations of the system [5-8]. For this purpose a detector simulation program, called HORUS, was developed in the context of one of the European XFEL detector development projects (AGIPD). This program was developed with two aims:

Detector simulation: in order to study the influence of each individual component on the overall detector performance, as well as the influence of various technological limits.

Science Simulation: evaluate the impact of the detector on the scientific experiments to be performed with the AGIPD detector and thus guide the decision process on necessary compromises between conflicting requirements, like pixel size and pixel functionality for instance.

First a description of the HORUS program will be given, this will be followed by two examples of its use.

\section{Description of the program}

HORUS (HPAD Output Response fUnction Simulator) is a detector simulation program simulating all the physical and electronic processes impacting the detective quantum efficiency of the detector. It was written using IDL (the Interactive Data Language). IDL is a software developed and 


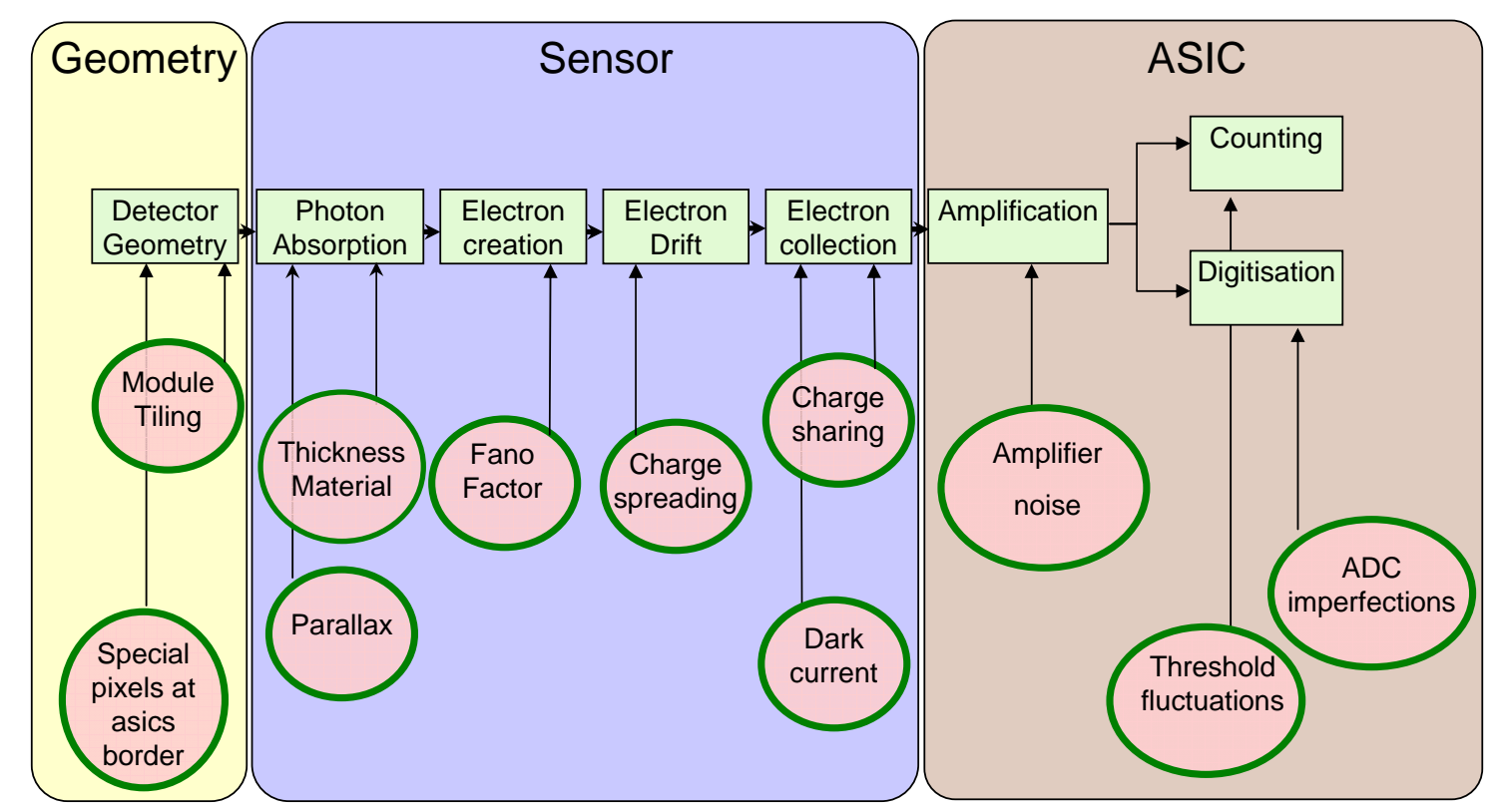

Figure 2. The HORUS simulation program structure.

maintained by the ITT Visual Information Solutions company [9]. Some functions make use of the IMSL library, which is part of the basic IDL license from version 7.1 on.

The program was written using a modular structure, following step by step the various physical and electrical processes involved in the signal detection process. Care was taken to allow easy modification of the models implemented or even complete replacement of modules.

It should also be understood that in the current version the models taken to describe the physical processes were purposely kept simple, and can be refined if necessary.

In particular, it was chosen not to make use of any of the classical Monte Carlo simulation tool like Geant4, Penelope or Fluka [10-12] in order to keep the tool easy to program, and the flexibility maximal. It is clear though that an interfacing to one of these programs could be very beneficial, especially if photons of energies higher than several $10 \mathrm{keV}$ would have to be simulated, since Auger and fluorescent photons, as well as multiple scattering of electrons can play a major role at these energy ranges.

The detector simulation program is part of the European XFEL AGIPD detector development program, and thus reflects the particular implementation of this detector, but HORUS can easily be adapted to any other Hybrid Pixel detector, or even to any other type of detector with greater modifications to the program. As stated before, the program can be used both to study the overall detector performance as function of various technological choices, and to simulate the degradation of any input image in order to study its impact on the scientific application.

The time of execution depends mostly on the number of photons in the input picture. Typically, a picture with a total of 500000 photons will be processed in less than three minutes on a standard Pentium D powered computer with $2 \mathrm{~GB}$ of memory.

In the following, first the part of HORUS common to all hybrid pixel detectors is presented (sensor and first stage amplifier simulation), then in a second part, the specific implementation of 
HORUS for the AGIPD is described. In the last part of the manuscript two examples of the use of HORUS are given, and its educational interest for young detector scientists is emphasized.

\subsection{Overall structure}

The program is an IDL function taking a picture as input argument, and returning an integer picture, with the same size as the detector. For example:

output $=$ horus (input) .

In this example, input would be a picture describing the photon distribution at the detector surface: each pixel value indicates the number of photons which will enter the detector in the corresponding detector pixel. If the input picture is not of an integer type, it is converted to a long format.

The input pictures can be obtained from either simulations of the future experiments, or, alternatively, be extrapolated from experimental data if the experimental conditions and overall background noise characteristics are to be included.

Therefore, the precise experimental conditions do not need to be known, but the distance of the detector to the sample must be defined to determine the local angle of the photons with respect to the detector surface, as well as the photon energy. Currently, all photons are assumed to have the same energy; if necessary, it is also very easy to modify the program so that the photon energy is computed randomly after any energy distribution function.

As the precise coordinates of the photons within a pixel are not known, each photon's precise position within the pixel is assigned randomly. In order to improve the precision of the simulation, it is possible to use oversampled input pictures where several pixels of the input picture are used to describe the intensity distribution within one detector pixel.

Global variables defined at the beginning of the program are used to set the detector parameters, such as the sensor thickness, the detector pixel size, the number of pixels per chip, the number of chips per sensors, the gap size between chips, etc. This way, almost any planar detector geometry can be described simply by changing these values.

As indicated in figure 2, the program structure reflects the process of detection and signal flow in a real detector:

- Data is removed form the input picture corresponding to the detector dead areas.

- Photon absorption, charge creation, and charge transport in the sensor.

Charge collection at the bottom of the sensor taking into account charge sharing between pixels.

- Charge amplification, and if necessary charge storage and digitization.

- Reconstruction of the image.

All these processes can be easily activated or deactivated either by modifying the source code, or more easily with the use of run-time options enabling the separate study of each of the detector components. the syntax becomes then:

output $=$ horus(input, /Boolean_option, parameter = parameter_value). 
Among other possibilities, the following subset of implemented options may be used as runtime boolean options:

no noise can be used to suppress any noise contribution in the detector. That is, sensor leakage, amplifier noise, analog storage cells leakage and charge injection, and ADC noise.

no electron conversion to suppress the sensor simulation, and study only the ASIC (Application Specific Integrated Circuit) behaviour. The number of photons is then simply converted into a number of electrons depending only on the sensor material and photon energy.

no dead area to make the simulation of a monolithic single detector without dead areas, and larger pixels.

ADU unit (Arbitrary Detector Unit) to study the actual detector response in ADUs, without the conversion into photons for the reconstructed picture.

The same way, among other the following simulation parameter values can be set:

photon energy $=\mathbf{X}$ can be set to override the default photon energy set at the beginning of the program. $X$ is float of unit keV.

oversampling $=\mathbf{n}$ can be used to define the oversampling factor, that is the number of pixels of the input picture used to describe the intensity distribution inside of one detector pixel. $n$ is an integer.

All these parameters are optional, and possess default values which are used if no value is passed to the function. They are mostly used to allow for an easy writing of scripts launching several simulations with various parameters. The implementation of a new parameter to control one of the simulation parameters is a trivial task.

The following subsections describe in greater detail each of the simulation steps listed above.

\subsection{Geometry}

Large hybrid pixel array detectors are constructed out of basic modules, consisting of monolithic sensors bump-bonded to multiple readout ASICs, and exhibit dead areas between the modules. In addition, the pixels between two ASICs on a sensor as well as the pixels at the edge of the sensor are larger than the nominal one. This has an impact on both the limited sampling frequency at these places, and the noise behaviour of these pixels because of locally higher leakage currents and higher intensities.

These effects are fully taken into account, first by summing the pixel values at these locations, and then by accounting for the higher sensor leakage values.

In practice, the program cuts the original pictures into modules representing the physical modules of the detector, and then processes them sequentially. The cutting is done according to the specific detector geometry and can leave a square central hole of any size, in multiples of one pixel. An example is given later in section 3.1, and figure 7.

Each module entity contains the information of its position relative to the whole detector, enabling a specific treatment of the photon absorption process down to the pixel level, in particular to treat parallax effects properly, and an easy reconstruction of the final picture from the modules. 


\subsection{Sensor simulation}

The simulation of the sensor behaviour is the most time consuming step of the simulation. A Monte Carlo like approach, in which each incoming photon is treated separately, is taken. In the current version no simulation of fluorescence photons, photon elastic scattering processes and electron scattering is performed. This is justified by the energy range ( 1 to several $\mathrm{keV}$ ) in which elastic and fluorescent cross sections are largely dominated by the photoelectric effect.

At the moment, all the implemented parameters correspond to typical Silicon sensors, as they can be found in most soft X-ray sensitive hybrid pixel detectors, but the simulation parameters can easily be changed to reflect other material characteristics like GaAs, $\mathrm{CdTe}, \mathrm{Ge}$, etc.

The sensor simulation model takes a two step model, in which the photon is absorbed somewhere along a straight line in the sensor, followed by gaussian spreading of the created electron cloud during transport through the sensor.

The distance $l$ travelled by the photon in the silicon sensor is computed randomly for each photon assuming a constant mass absorption coefficient $\mu$ :

$$
\frac{d I}{d l}=\mu d l
$$

leading to an exponential absorption profile:

$$
I(z)=I_{0} \cdot \exp (-\mu l) .
$$

The actual implementation of the calculus of $l$ is thus:

$$
l=-1 / \mu \cdot \log (\text { random })
$$

where 1 has the same unit as $1 / \mu$ and random is a number randomly generated in the range $] 0,1[$, after a uniform law.

As explained previously, the 3D coordinates of the photon absorption point $\vec{a}=\left(x_{a}, y_{a}, z_{a}\right)$ are computed from a point randomly chosen at the pixel surface, corrected from the projection of $l$ on each of the $\vec{x}, \vec{y}, \vec{z}$ directions (see figure 3). For example $x_{a}=X+l \cdot \cos \left(\theta_{\mathrm{p}}\right) \cdot \operatorname{cotan}\left(\chi_{\mathrm{p}}\right)$ where $\mathrm{X}$ is the random point $\mathrm{x}$ coordinate, $\theta_{\mathrm{p}}$ is the angle of the photon direction with its projection $l_{\mathrm{p}}$ on the sensor surface and $\chi$ is the angle of $l_{\mathrm{p}}$ with the $\vec{x}$ direction.

If $z_{a}$ is larger than the sensor thickness, the photon is undetected. Similarly, if one of the photon absorption coordinates $x_{a}$ or $y_{a}$ is found to be beyond the sensor limits, the photon is undetected.

The process of conversion of the incoming photon into electrons is assumed to be at a point, and the number of created electrons is computed randomly assuming Poisson statistics of reduced variance

$$
\sigma_{\text {reduced }}=f \cdot \sigma_{\text {poisson }}
$$

where $f$ is the fano factor, assumed to have a value of $f=0.1$ in the case of silicon, $\sigma_{\text {reduced }}$ and $\sigma_{\text {poisson }}$ are the effective and expected variances if the process would be purely random $[13,14]$. The mean electron ionizing energy is assumed to be $3.65 \mathrm{eV} /$ electron and all the photon energy is converted into electrons, i.e. $\sigma_{\text {poisson }}=\sqrt{\frac{E_{\text {photons }}}{3.65}}$, with $E_{\text {photons }}$ the energy of the incoming photons in $\mathrm{eV}$. 


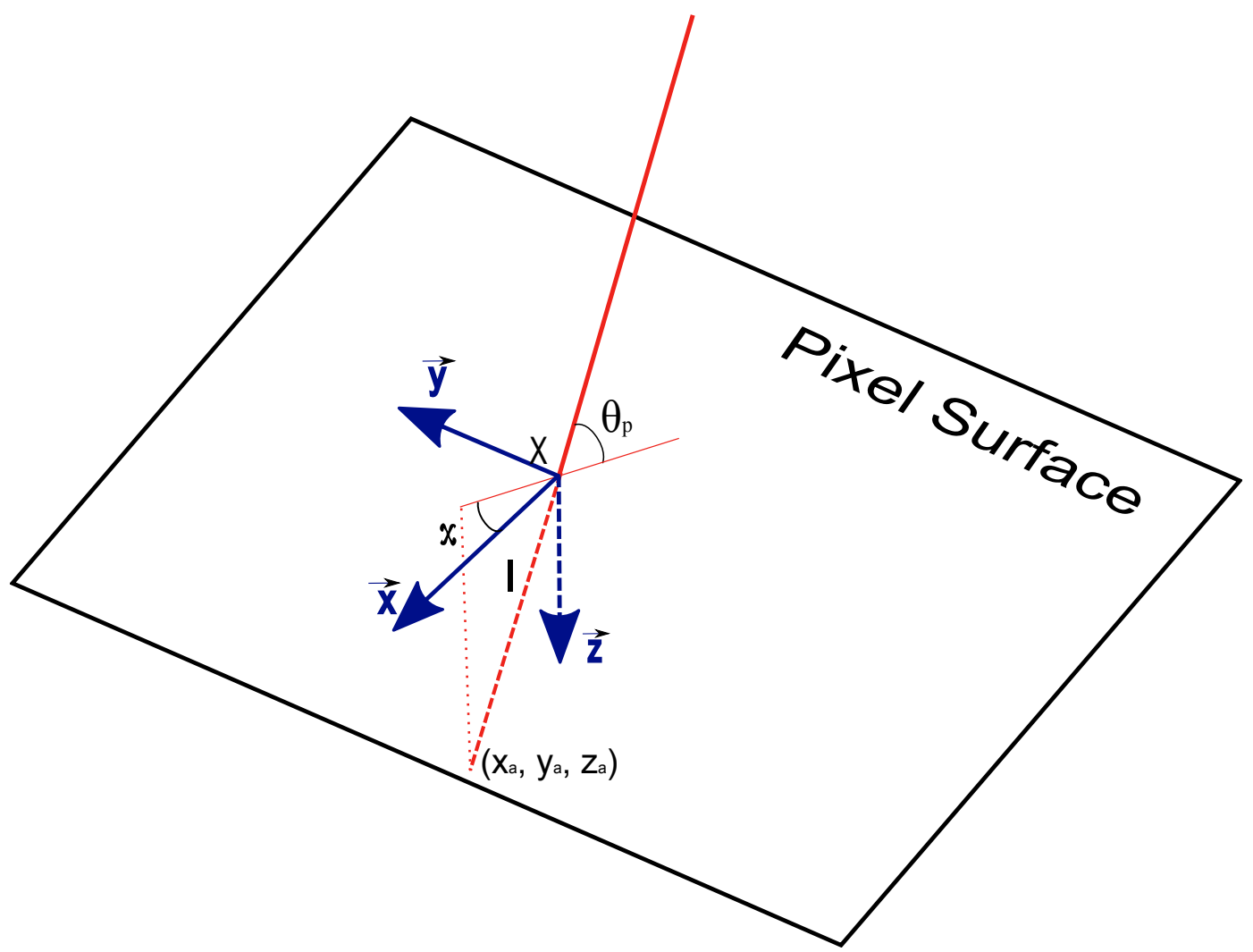

Figure 3. Local coordinates definition for calculus of the path length projection values.

The spreading of the electron cloud is then computed assuming a 2D circular Gaussian distribution function of spread $\sigma=\sqrt{2 D t}$, where $D$ is the diffusion coefficient of the sensor material, and $t$ the drift time of the electrons to the bottom of the sensor [13, 14]. $t$ is determined from the absorption coordinates $\vec{a}$, the saturation velocity of the electrons (or alternatively of the holes) in the sensor material, and the sensor thickness; this is a good approximation only in the case of fully depleted sensors, and in the absence of electron-hole plasma [15].

The occurrence of electron-hole plasma is a complicated process which can happen when high photon fluxes result in charge carrier densities higher than the intrinsic doping level of the material and its consequences are not yet completely understood. The created charge cloud then becomes insensitive to the applied bias across the sensor and the charges do not drift until the charge cloud has diffusively expanded enough for the drift field to take over again. This results in an increased spreading of the signal, and a longer collection time. The process is still under both experimental and theoretical study but it is expected that the effect can be neglected in most experiments at the X-ray FELs [15].

Alternatively, the spread value $\sigma$ can be computed from the detailed simulations performed by R.F.Fowler et al., of which the details can be found in [16].

The proportion of the electron cloud in the central pixel where the photon is absorbed and in each of the 8 neighbouring pixels is computed from the Gaussian cumulative distribution func- 


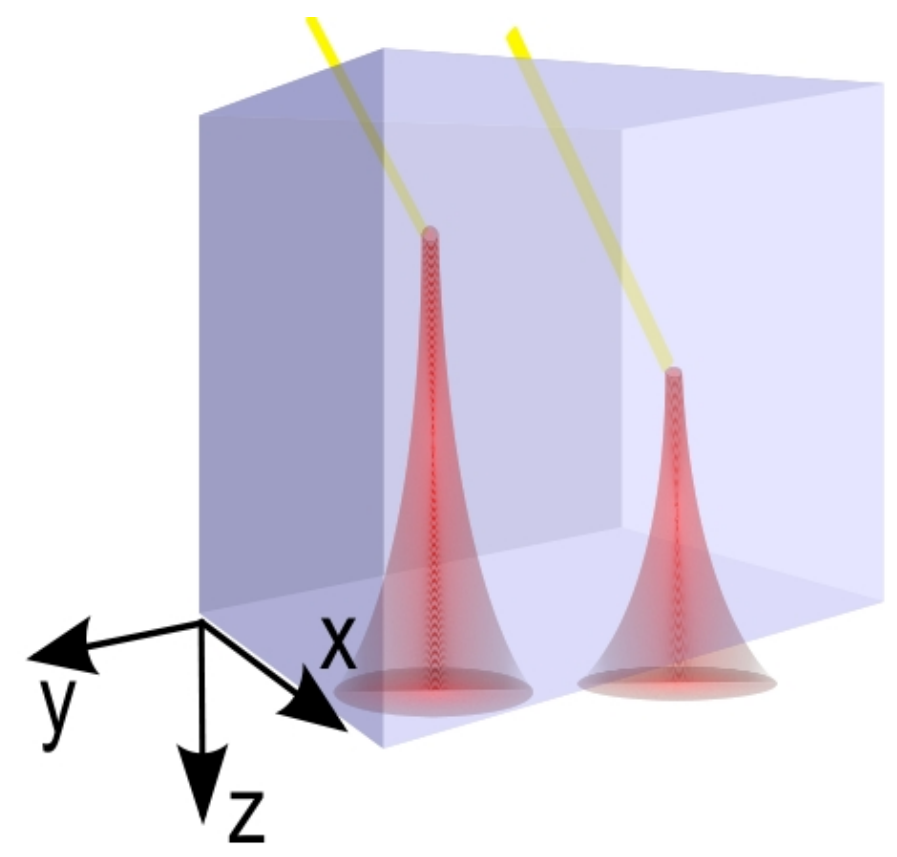

Figure 4. Sensor Simulation: Absorption of photons (yellow) and charge spreading simulation (red).

tion using a built-in IDL function. The actual implementation of this calculus makes use of the cumulative distribution function:

$$
C D F(X, Y)=\int_{-\infty}^{X} \int_{-\infty}^{Y} P D F(x, y) d x d y
$$

where $P D F$ is the bivariate Gaussian probability distribution function for uncorrelated variables

$$
P D F(x, y)=\frac{1}{2 \pi \sigma^{2}} \exp \left(-\frac{x^{2}+y^{2}}{2 \sigma^{2}}\right) .
$$

Since the integration in the cumulative distribution formula is performed from $-\infty$ to $X$ and $-\infty$ to $Y$, the contribution Contr. of the cloud in one pixel is (see figure 5):

$$
\begin{aligned}
\text { Contr. } & =C D F\left(D_{x}+S_{x}, D_{y}+S_{y}\right)-C D F\left(D_{x}+S_{x}, D_{y}\right)-C D F\left(D_{x}, D_{y}+S_{y}\right)+C D F\left(D_{x}, D_{y}\right) \\
& =\int_{0}^{S_{x}} \int_{0}^{S_{y}} \operatorname{PDF}\left(D_{x}, D_{y}\right) d x d y
\end{aligned}
$$

where $D_{x}$ and $D_{y}$ are the distances of the absorption point $x_{a}$ to the pixel edges in the $\vec{x}$ and $\vec{y}$ directions, and $S_{x}$ and $S_{y}$ are the pixel sizes in the respective directions.

The last part of the sensor simulation concerns the sensor leakage. Modern readout electronic designs include a sensor leakage compensation mechanism. Careful calibration of these mechanisms ensures a small but non zero signal contribution from this process, mainly due to its statistical origin.

A simple model of sensor leakage behaviour after compensation is included in HORUS: the residual leakage is considered to be random and to follow a Poissonian behaviour, of centre value a fraction of what it would be without compensation mechanism. The actual value can be extracted from measurements. 


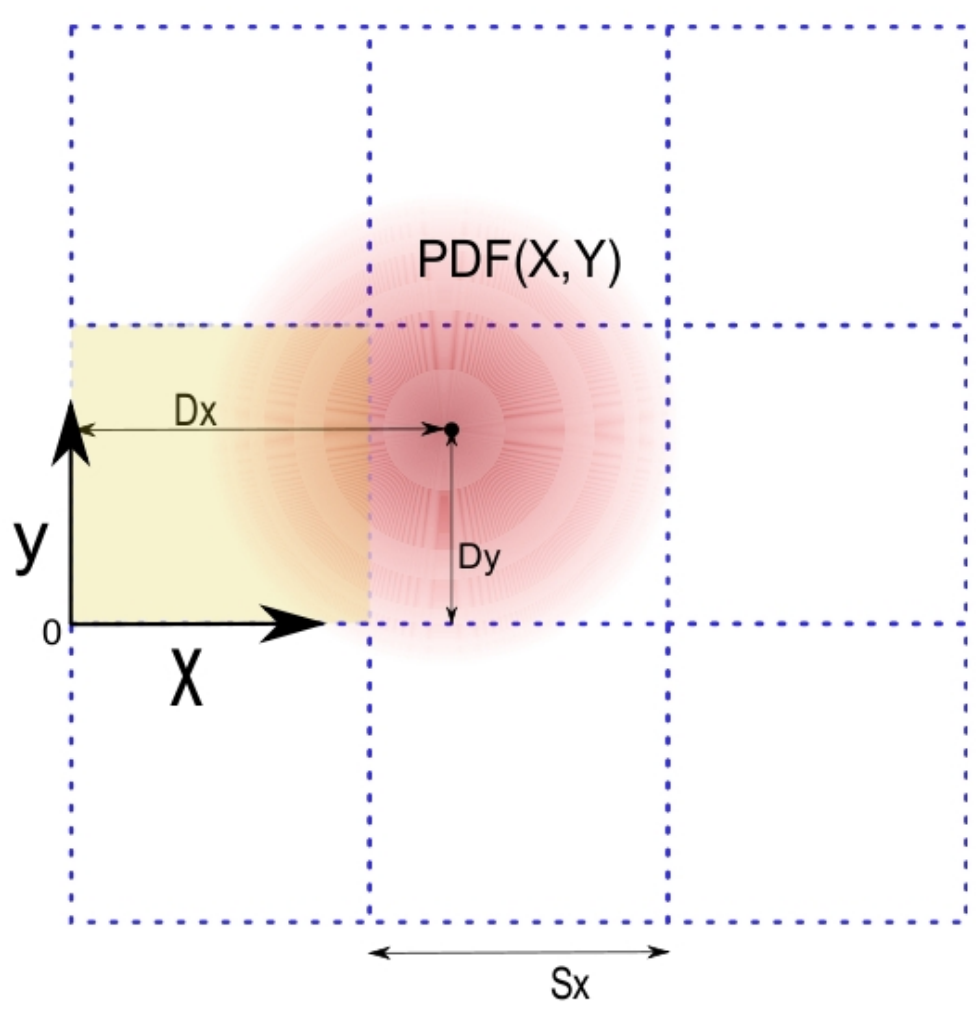

Figure 5. Coordinate system for the computation of the electron cloud spreading in the pixel directly left to the central one.

\subsection{Readout electronics simulation}

The first step of the ASIC and electronics simulation is the simulation of the first amplifier stage. The noise contribution from the amplifier is assumed to be the Gaussian distributed equivalent noise charge spread (as found in [14])

$$
Q_{\mathrm{n}}^{2}=\frac{\exp (2)}{8}\left[\left(2 e I_{\mathrm{d}}+\frac{4 k T}{R_{\mathrm{p}}}+i_{\mathrm{na}}^{2}\right) \tau+\left(4 k T R_{\mathrm{s}}+e_{\mathrm{na}}^{2}\right) \frac{C^{2}}{\tau}+4 A_{f} C^{2}\right],
$$

with $Q_{\mathrm{n}}$ the equivalent noise charge, $\frac{\exp (2)}{8}$ the normalization factor of $Q_{\mathrm{n}}$ to the signal gain, $e$ the electron charge, $I_{\mathrm{d}}$ the average (i.e. leakage) detector current, $k$ the Boltzman constant, $T$ the temperature, $R_{\mathrm{p}}$ the equivalent parallel resistance, $i_{\mathrm{na}}^{2}$ the equivalent input current noise, $\tau$ the shaping time, $R_{\mathrm{S}}$ the equivalent input series resistance, $e_{\mathrm{na}}^{2}$ the equivalent input noise voltage, $C$ the input capacitance, and $A_{f}$ the low frequency "1/f" noise power density. This contribution is added to the recorded signal, and is computed randomly for each individual pixel.

Transient noise sources are not yet included in the simulation.

The remaining detection chain is then very detector specific:

- In the case of a counting detector, for systems like the Pilatus or the Medipix2 readout chip for example, it is enough to set a threshold value to count or not the signal as a photon. Pixel-to-pixel threshold dispersions can easily be incorporated. In the case of more complex 
systems, such as the Medipix3 readout chip, which has some charge sharing compensation mechanisms, a more complex model is needed.

- In the case of an integrating detector, at least the ADC (analogue to digital converter) behaviour has to be simulated. Also, additional features such as dynamic gain switching and in-pixel analogue storage pipelines must be incorporated in the simulation tool.

In the second case, HORUS also offers the possibility to perform the photon reconstruction from the digitized signal: the reconstruction of the detected photons in each pixel is performed from the theoretical gain factor $\varphi$ of the detector:

$$
\varphi=\frac{\varepsilon \cdot \Gamma}{\Delta},
$$

with $\varepsilon$ the mean number of electrons produced, $\Gamma$ the amplifier gain, and $\Delta$ the ADC resolution assuming that the full ADC dynamic range is used: $\Delta=\frac{1}{2^{n}} \cdot D R$ for a $\mathrm{n}$ bits $\mathrm{ADC}$, and a signal dynamic range DR.

Any pixel having a signal $\left(m-\frac{1}{2}\right) \cdot \varphi \leq$ Signal $\leq\left(m+\frac{1}{2}\right) \cdot \varphi$ will be considered as having recorded $m$ photons. More complicated schemes taking charge sharing into account can also be implemented.

\section{Example of uses of HORUS: Study of the AGIPD}

One of the main motivations for the development of HORUS is the study of the AGIPD behaviour as a complete system, to identify key components and limitations, to evaluate their impact on the experimental output, and to help achieve the best compromise between conflicting requirements. There are several ways of doing this:

- Study of the statistical response characteristics, by studying the detector response spreading as a function of the various noise contributors: amplifier noise, charge sharing effect, etc.

- Study the detector response to the input images, either photon by photon, or statistically. The recorded image can then be compared to the input image and the impact of detector imperfections on the scientific output can be studied.

First a description of the AGIPD will be given. This is followed by an example of a study of the statistical response of the detector, illustrating how HORUS can be used to evaluate the detector response spreading as a function of various parameters, and for various conditions. Then, an illustration of a science simulation study is presented. This study is made by looking at the degradation of a prototypical image, corresponding to a typical future XFEL experiment. Finally, a short description of the educational interest of HORUS is given.

\subsection{Description of the AGIPD and its simulation}

\subsubsection{The AGIPD}

The Adaptive Gain Integrating Pixel Detector (ex XFEL-HPAD), is one response to the need of large $2 \mathrm{D}$ detectors, able to cope with the very high repetition rate of $5 \mathrm{MHz}$, as well as with the 


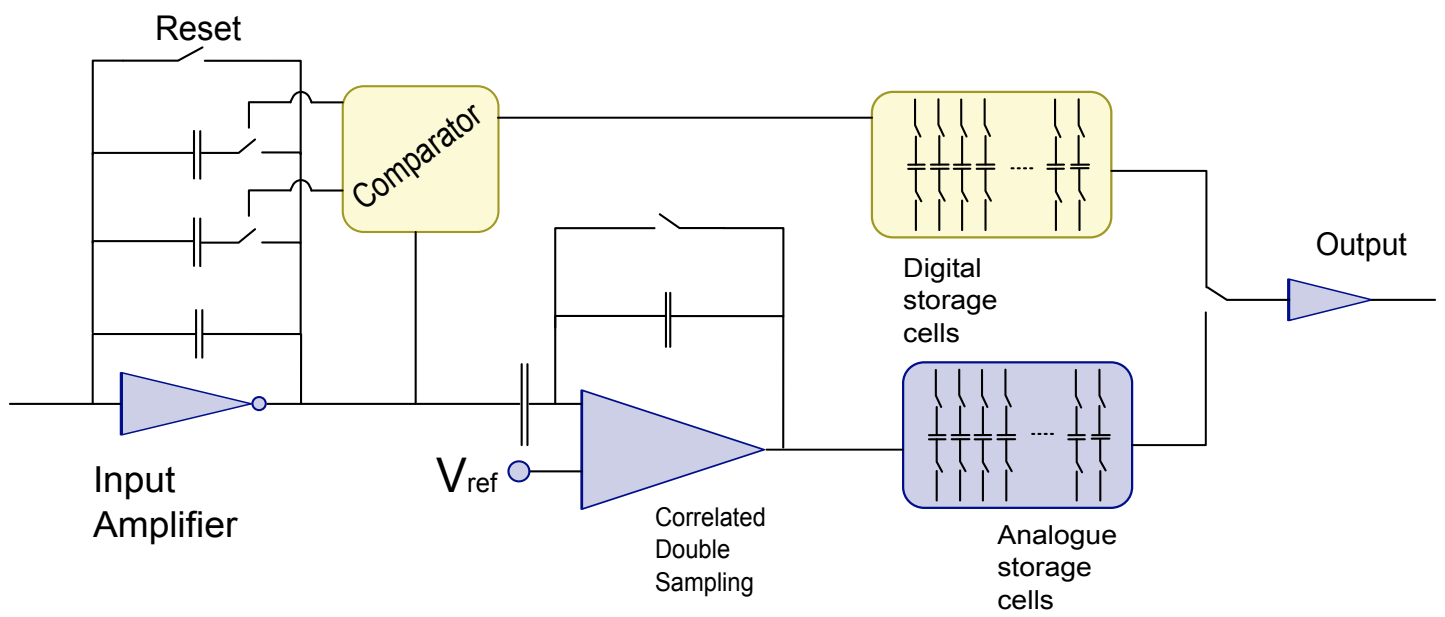

Figure 6. The AGIPD readout ASIC layout.

required dynamic range ranging from single photons to $10^{4}$ photons of $12 \mathrm{keV}$ per pixel and per pulse. In addition, the detectors should be sufficiently radiation hard to withstand the expected high doses.

The AGIPD detector will feature advanced in-pixel electronics, such as a dynamic gain switching amplifier system, and an analogue storage pipeline. It is based on the hybrid technology, in which a silicon sensor is bump bonded to readout ASICs, thus enabling the separate optimizations of the signal absorption and processing functions.

The ASIC will operate in charge integration mode, where the amplifier response is proportional to the input signal [13]. Figure 6 gives a schematic of the pixel electronics of the ASIC. The high dynamic range is achieved by the addition of two more feedback capacitors to the charge sensitive amplifier which can be dynamically activated, thus enabling a self-adjustment of the gain to the signal. Each pixel will also contain an analogue pipeline, able to store several 100 pictures at the maximum speed of 5MHz. The readout of the pictures will be done between the XFEL pulse trains.

The basic chip unit (the ASIC) will have a size of $64 x 64$ pixels of $200 \mu m x 200 \mu m$, and $8 x 2$ of these chips will be bump bonded together to a single $500 \mu \mathrm{m}$ or $700 \mu \mathrm{m}$ thick silicon sensor. In order to avoid dead areas between the chips, the effective pixel sizes of the outer pixels will be made twice (at borders) or four times (in corners) larger. 16 of these modules will be mounted together in order to form the full $1 \mathrm{kx} 1 \mathrm{k}$ pixel detector, with the option for a later extension to $2 \mathrm{kx} 2 \mathrm{k}$ pixels or larger. Figure 7 depicts the overall detector, and shows the variable size central hole, through which the direct XFEL beam and the sample forward scattered signal will be transported.

The analogue to digital conversion will be done on a separate readout card, with a 14bit ADC, and a "train builder" will do the reconstruction of the images stored in the ASICs.

\subsubsection{AGIPD specific simulation models in HORUS}

The AGIPD specific geometry and sensor simulations follow exactly the process described previously. 


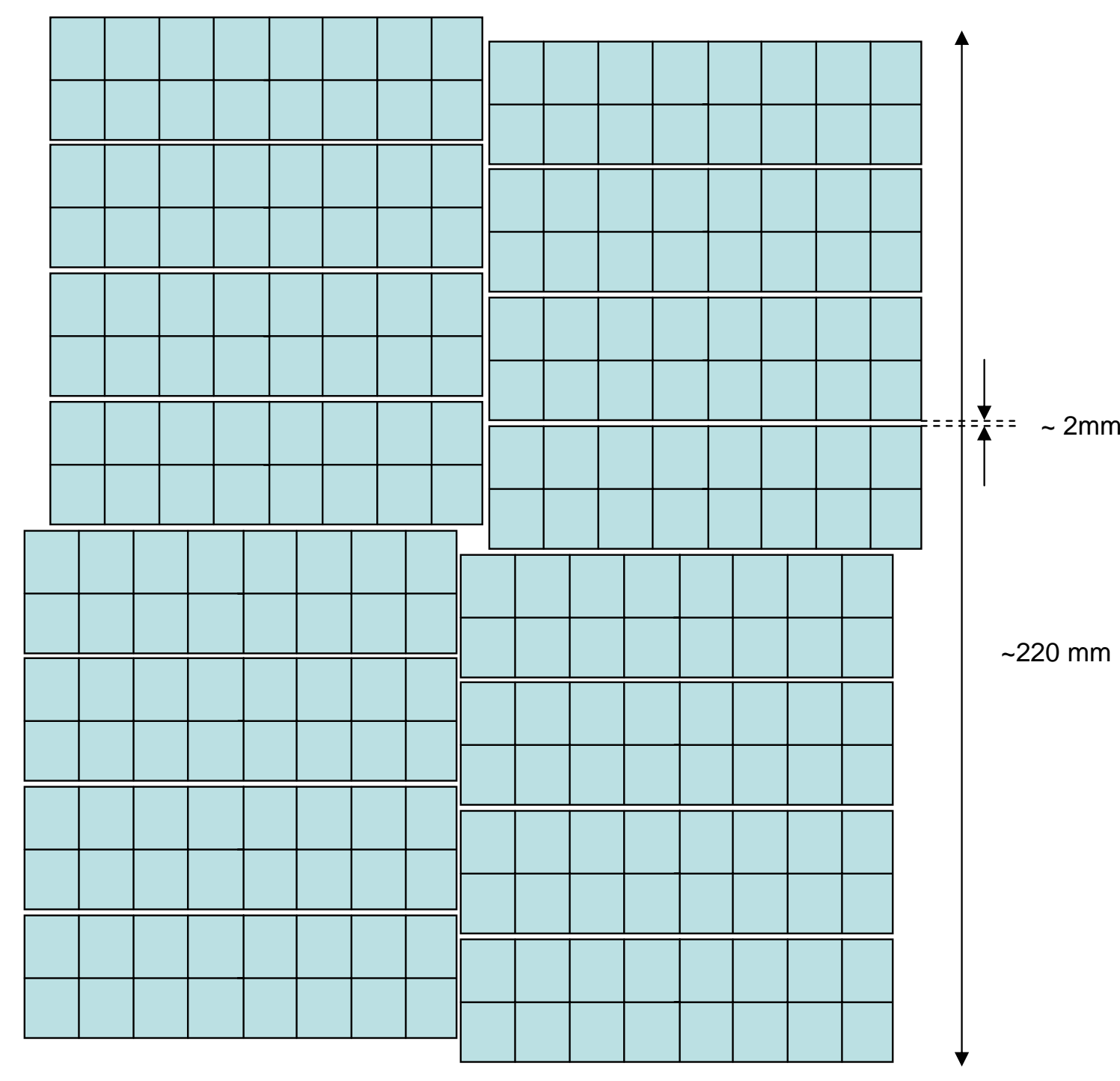

Figure 7. The AGIPD layout.

To simulate the AGIPD ASIC properly, it is necessary to add a certain number of elements to the generic part of the simulation program, in order to take the gain switching mechanism, the analogue pipeline behaviour, and the analogue to digital converter (ADC) imperfections into account.

The dynamic gain switching is simulated by changing the gain factor for each pixel according to the number of collected electrons, computed as explained in the previous section. The gain and noise characteristics of the amplifier are extracted from detailed simulations down to the transistor level rather than from the general formula (2.9) given in section 2.4.

The digital pipeline keeping track of the used gain for each pixel is also simulated, in order to allow for the correct reconstruction of the pixel intensity.

The analogue pipeline imperfections are simulated assuming two contributions to the noise: if $S$ is the signal after the input amplifier, the readout signal has the form $S_{\mathrm{ADC}}=A\left(S+C_{1}\right)+C_{2}$ with:

- $C_{1}$ and $C_{2}$ contributions to the recorded signal, in order to take into account for possible 
charge injections by the switches used to route the signal to the proper storage cell, and for readout. $C_{1}$ and $C_{2}$ are random contributions following a Gaussian distribution function of centre 0 .

- $A$ is a proportionality factor, enabling the simulation of possible leakage currents in the capacitors or switches of the storage cell. A worst case scenario of a long storage time $(100 \mathrm{~ms})$ is always assumed. $A$ is computed from a gaussian distribution, of centre 1 .

In practice, the formula $S_{A D C}=S \cdot A+C$ is used, where $C^{2}=\left(A \cdot C_{1}\right)^{2}+C_{2}^{2}$, since the convolution of two Gaussian distributions is a Gaussian distribution. The spread values of the distributions describing $A$ and $C$, are the result of measurements on test structures. In the future, an improved model may be implemented to take into account a possible non linear behaviour of the storage cells.

Subsequently the digitization of the signal is performed. The 14bit ADC forssen is the AD9252 from Analog Devices. It is assumed to be perfectly linear in the region of operation, and a Gaussian digitization error of 4.6 LSB (least significant bit) is assumed during the digitization. The full 14bit dynamic range is used.

In the Final step, the image is reconstructed by combining the gain and digitization information.

\subsection{Study of the AGIPD statistical response}

To study the detector statistical response, it is possible to analyze the simulation result just after the digitization step. This corresponds to the signal gain noted $\varphi$ in 2.4.

The signal's unit is then specific to the system, and will be called here ADU units. A perfect detector would return only one signal value, corresponding to the gain value $\varphi(I)$. But in a real detector the various imperfections result in a certain distribution $\Phi(I)$ of the detector gain $\varphi(I)$, and a corresponding spread of the returned signal value in ADU.

For example, one can study the detector response in the case of intensities of 1 and 10 photons per pixel.

To perform this study, we created an artificial input image. Since the local angle of the photon direction with the surface has an impact on the detection process, this image has to be small enough to limit variations of this effect over the image. In addition, neighbouring pixels have an impact on each other via charge sharing, and parallax, so the pixels containing intensities, from hereon called "hot" pixels, must be well separated, to avoid any mutual influence.

Two pictures were created with a set of pixels having an intensity of 1 and 10 photons respectively, and with a minimum space between these hot pixels of 3 pixels.

The response frequency diagrams corresponding to both cases $I=1$ and $I=10$ are depicted in figure 8. The response frequency diagram is simply the response diagram normalized to the total number of hot pixels such that the sum of all displayed frequencies is 1 . This enables an easy comparison of the values in various cases.

Each diagram gives the normalized relative frequency as function of the detector output signal. The response dispersion is a direct measure of the detector gain distribution $\Phi(I)$, as each signal value corresponds to one specific gain value $\varphi$.

The left diagram corresponds to the case $I=1$. It exhibits two peaks; the right peak is the actual detector response to the 1 photon intensity. The left peak corresponds to the many pixels 

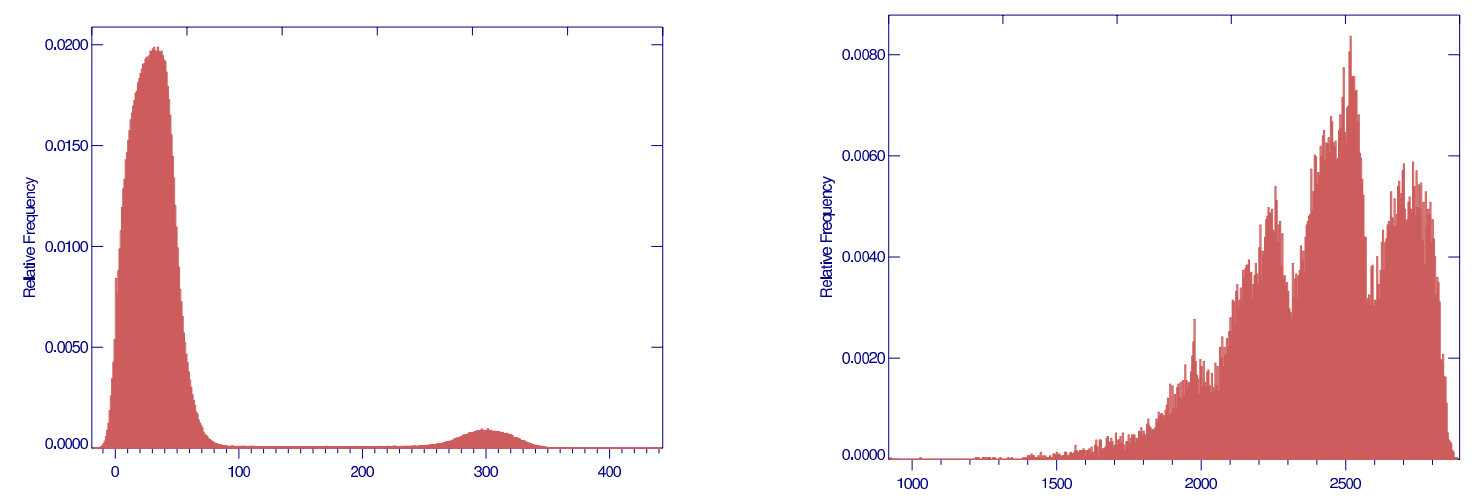

Figure 8. Detector gain frequency diagram in the case of an intensity of 1: $\varphi(I=1)$ (left) and 10: $\varphi(I=10)$ (right) photons.

with no photon, and only internal noise as well as signal received from neighbouring hot pixels via charge sharing. The right peak is showing the expected response spreading, and can be described in first approximation by a Gaussian; the Gaussian spread $\sigma$ of the peak is a measure of the response spreading of the whole detector system at this intensity, and is the result of the various imperfections of the detection process, such as spreading of the charge cloud during charge transport in the sensor, the noisy amplification of the signal, and the non perfect digitization of it by the ADC.

The relative sizes (areas) of the two peaks reflect the relative proportions of the pixels with 0 or 1 photon intensities in the picture. Since they are more empty pixels, than pixel with 1 photon, the left peak is bigger.

One can note that the integral of the right peak is $\int($ peak $) \simeq 0.9 \cdot f_{\text {hot pixels }}$, with $f_{\text {hot pixels }}$ the ratio of the hot pixels to the total number of pixels. This means that only $90 \%$ of the incident photons were detected, which is consistent with the expected quantum efficiency of $90 \%$ at $12 \mathrm{keV}$ energy for the AGIPD with a $500 \mu m$ thick silicon sensor.

The interpretation of the right diagram of figure 8 (case $I=10$ ) is less straight forward since it has more than one Gaussian shaped peaks with various centres. The left most peak arising from the pixels with no intensity is not displayed for clarity reasons. This behaviour of the detector response can be explained by both the limited stopping power of the silicon sensor and by the parallax effect.

As in the case of $I=1$, the limited stopping power of the $500 \mu \mathrm{m}$ thick sensor results in $\sim 10 \%$ of the incoming photons passing through without being detected, and we have once again $\int($ peaks $) \simeq 0.9 \cdot f_{\text {hot pixels }}$, if $\int($ peaks $)$ is the integral over all the peaks.

The presence of several peaks indicates that some of the pixels which received 10 photons, actually detected less photons. The right most peak corresponds to exactly 10 photons recorded by the pixel. The last but one peak corresponds to only 9 photons being detected, etc.

This is expected since the process of absorption of each of the photons is independent of the absorption of the other photons, so each photon has a probability of $90 \%$ of being absorbed and detected, and this trial is repeated 10 times.

As a result, the relative magnitude of the various peaks is described by the binomial mass 


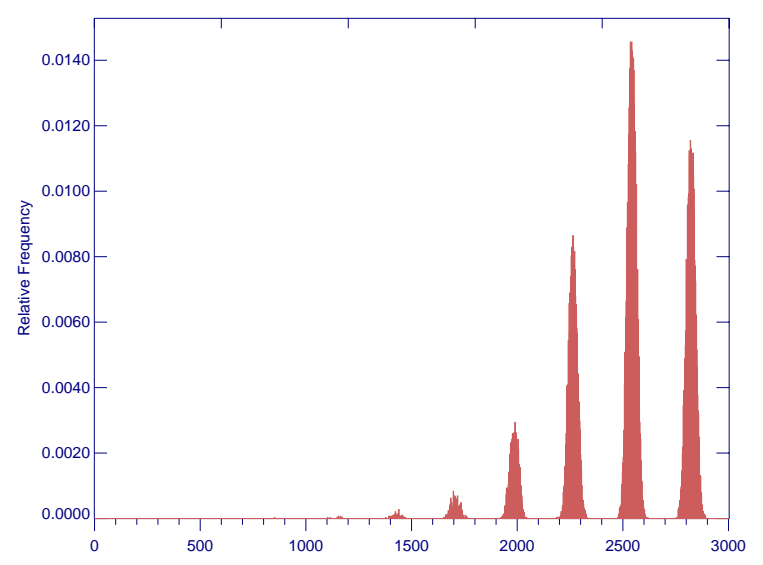

Figure 9. Detector response distribution (in ADUs) for the case of 10 incident photons if no charge sharing occurs in the pixels.

distribution function $\left(\frac{n}{k}\right) p^{k}(1-p)^{n-k}$ with $\left(\frac{n}{k}\right)=\frac{n !}{k !(n-k) !}$ the binomial coefficient. So in this particular example, the parameters values are $p=Q E=0.9, n=10$ photons, and $k=0,1,2, \ldots, n$. Each peak corresponds to one particular value of $k$, the number of photons being detected.

As expected, the highest peak in the diagram is the last but one on the right. It corresponds to the detection of 9 photons, which is the mean value expected for a detector of $90 \%$ quantum efficiency, in the case of 10 incident photons. This is also the expected mean of the binomial distribution function: mean $=n \cdot p$, and corresponds to the intuition that out of 10 photons, only 9 are actually detected if the detector quantum efficiency is $90 \%$.

The parallax effect, which results in certain photons being absorbed in a pixel different from the one in which they initially entered has a similar effect since actually less photons are detected in the pixel. This effect can be studied with HORUS by using a larger pictures in which the hot pixels are not at the centre, but are spread on a circle corresponding to a certain angle of the photons with the detector surface normal.

The width of the peaks also calls for an explanation: it is mostly due to the charge sharing effect which results in only a fraction of the charges created at the pixel border being collected in the pixel.

Finally, it is interesting to compare in picture 8 the detector response value for 1 and for 10 photons (right most peaks in left and right picture, respectively). While the detector returns a value of $\sim 300$ ADUs for a 1 photon intensity, it only returns a value of $\sim 2700$ ADUs in the case of a 10 photons intensity, indicating that the gain $\varphi$ of the detector is not strictly linear. This effect is simply due to the noise models which include both a fixe and an intensity variable contribution to the signal (see section 3.1.2), thus leading to a non linear behaviour of the measured signal.

If charge sharing is neglected, the width of the peaks is a lot smaller. For comparison, figure 9 gives the same diagram as in figure 8 when no charge sharing is simulated, i.e. all the charge deposited in one pixel is collected in this pixel. The remaining width is the result of the other imperfections, like amplifier noise, ADC noise, charge loss in the analogue pipeline, etc.

Activating and deactivating each of these processes enables to study very easily their relative importance, as well as their mutual impacts on each other. 


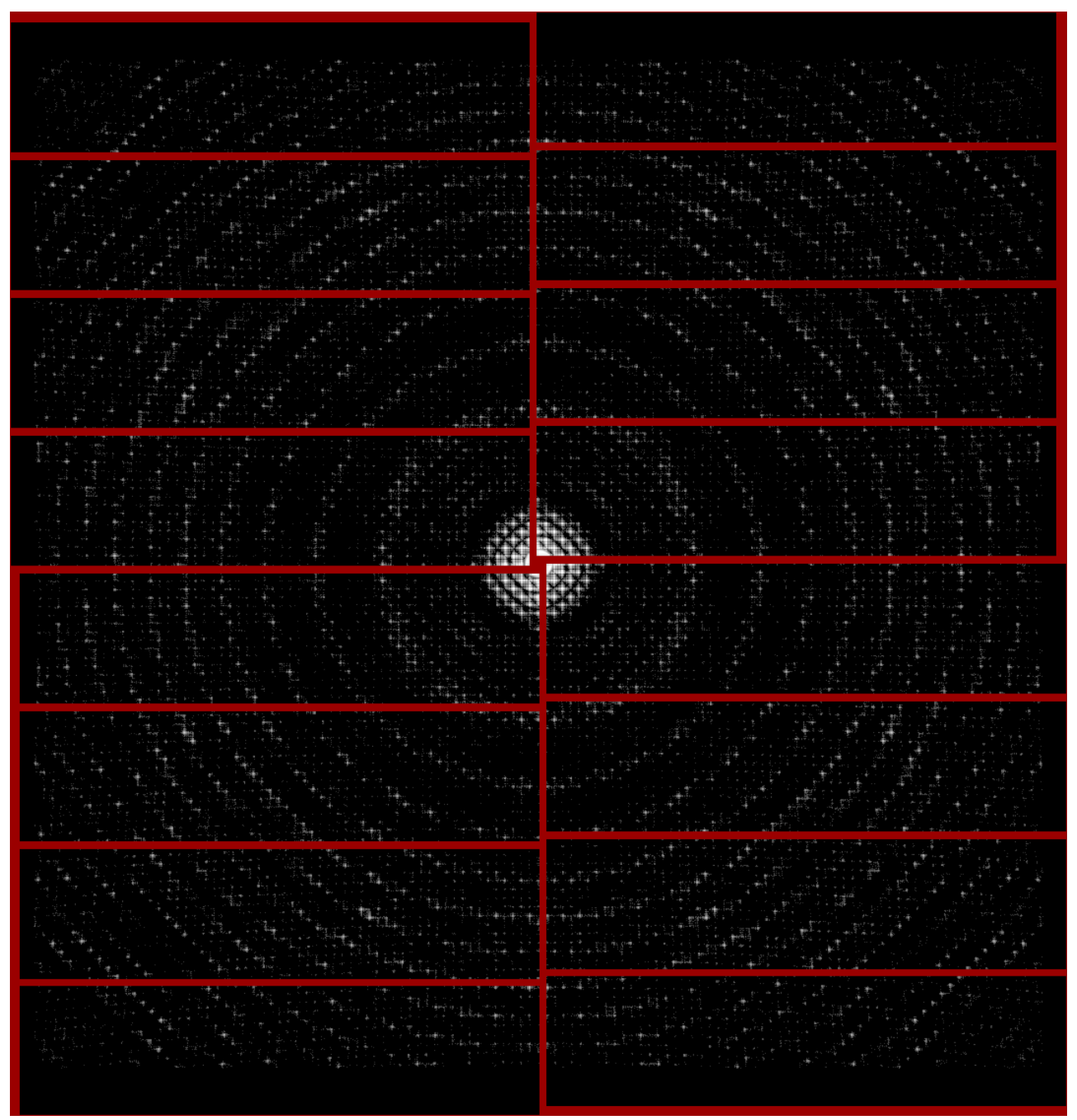

Figure 10. Diffraction pattern of a $5 \times 5 \times 5$ crystal units large ferritin crystal after simulation in HORUS (log scale).

\subsection{Science simulation: study of the image degradation}

As an example of an image degradation, a short study of the AGIPD detector response to a simulated small protein molecule crystal diffraction pattern is given.

Such diffraction patterns enable the reconstruction of the full protein molecular structure, if the signal to noise ratio is good enough. So performing the reconstruction of the molecule structure from the degraded diffraction pattern, as simulated by HORUS, enables the evaluation of the detector's impact on the reconstruction process.

The diffraction pattern was calculated from a known system: a small crystal of the human ferritin biomolecule ${ }^{1}$. The crystal is $5 \times 5 \times 5$ unit cells large, with 2 molecules per unit cell, giving a total of 250 molecules.

\footnotetext{
${ }^{1}$ The data are courtesy of Franz Pfeiffer
} 


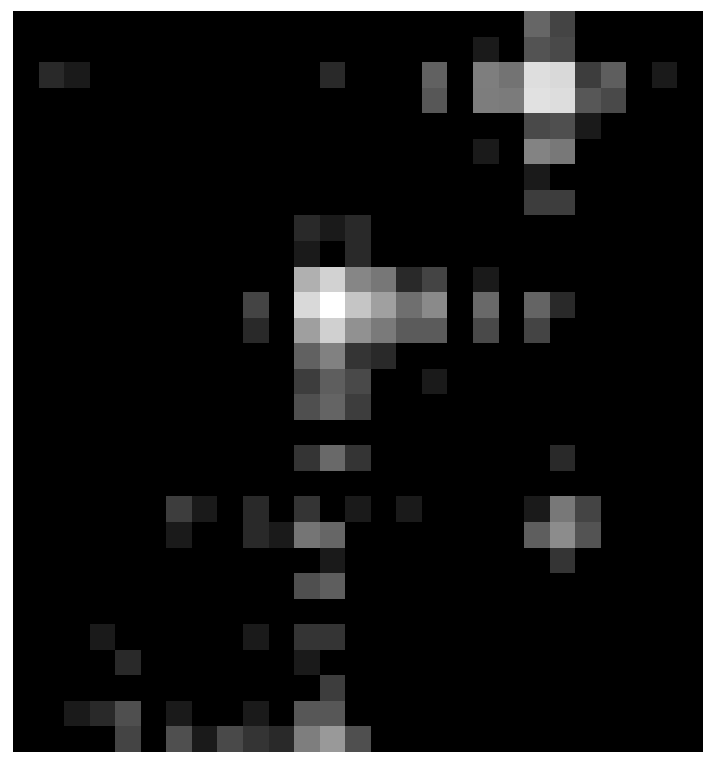

Figure 11. Local peak, located in the top left corner of the overall image.

The parameters taken are those of the SASE 1 beamline of the European XFEL and typical of an idealized XFEL: the beam is assumed to be fully coherent, with an intensity of $10^{12} 12 \mathrm{keV}$ photons/shot, focused down to a Gaussian spot of $100 \mathrm{~nm}$ full-width-half-maximum (FWHM). The detector-sample distance is $300 \mathrm{~mm}$, and the detector is assumed to be a "perfect" $1024 \times 1024$ pixel detector, with a pixel size of $200 \mu \mathrm{m}$.

An analysis of the diffraction pattern reveals that some pixels, in particular those very close to the forward direction, will receive extremely high intensities: in excess of $10^{9}$ photons per pixel. Since the maximum dynamic range of the detector developed for the European XFEL is less than $10^{5}$ photons per pixel per pulse, and since at these high intensities strong electron-hole plasma effects [15] become important, are currently not taken into account by HORUS, any pixel intensity above $10^{5}$ photons is reduced to $10^{5}$ photons.

The output picture, was computed in $\approx 10$ minutes, and the result is depicted in figure 10 (log scale). The detector dead areas are displayed in red.

To illustrate the local degradation of the picture, figure 11 shows a magnification of a peak in the top left corner.

A difference map of the original input picture and the picture returned by HORUS at the same location is shown in figure 12. Pixels in blue correspond to a deficit in the number of detected photons, and pixels in red to an excess with respect to the original image. The white pixels are those where intensities in both pictures are equal.

The difference map shows that many photons are missing at the place of the original peak, while many excess photons are in pixels at larger radii with respect to the peak, i.e. in the local photon direction. This highlights the effect of the parallax, which degrades the detector resolution.

For this peak there is an $8 \%$ deficit of photons in the recorded image when compared to the original, which is expected from the limited absorption of the sensor at this energy and incident angle. It is interesting to note, that this proportion changes with the distance to the centre of the 


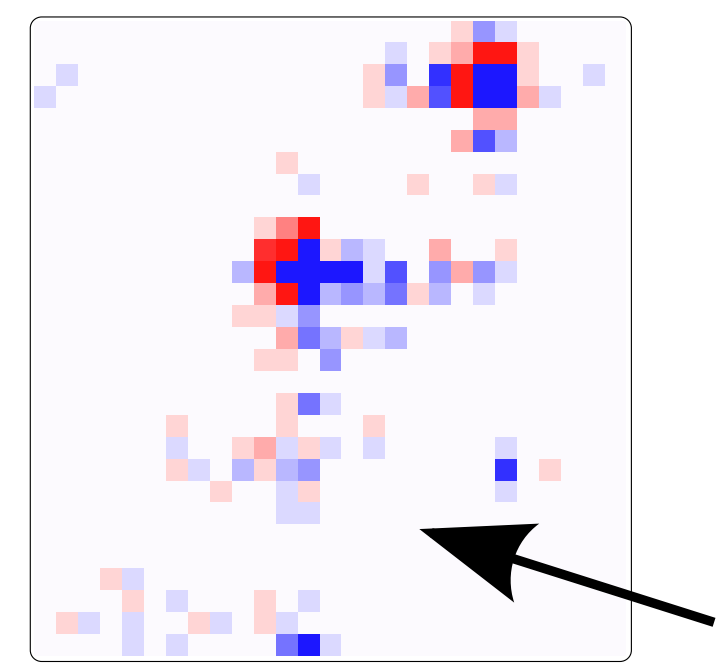

Figure 12. Difference map of the original picture and the HORUS output image at the peak position. The arrow indicates the local photons direction.

considered pixels, as expected from the increased projected sensor thickness at larger angles.

The possibility to compute such pictures makes it very easy to study the impact of detector imperfections on the experimental outcome. This is especially true for the experimental techniques making use of reconstruction algorithms, such as the coherent diffraction imaging techniques, which implies the use of phase retrieval algorithms. It is also possible to use these images to study the degradation of the signal to noise ratio in the case of experimental techniques such as X-ray Photon Correlation spectroscopy, which are based on a statistical analysis of the fluctuations in the speckle intensities.

More generally, the computation of such images is a powerful tool to evaluate the expected Detective Quantum Efficiency $D Q E=\frac{\text { Signal }_{\text {out }} / \text { Noise }_{\text {out }}}{\text { Signal }_{\text {in }} / \text { Noise }_{\text {in }}}$ of the detector, as one can simulate any procedure used to measure it on real systems. A similar work was already done with HORUS and the results were presented in [8].

\subsection{Educational aspect of HORUS}

Finally, the authors would like to emphasis the educational aspects of HORUS.

By deactivating any step of the detection process, and replacing it with an idealized behaviour, it is possible to isolate certain effects, which are normally hard to identify when studying a real system. Indeed, when evaluating a real system, the statistical fluctuations of the measured signal are the result of both the detector response characteristics, and of the intrinsic input signal fluctuations characteristics, which are in general described by Poisson distribution law.

So any process of the detection chain, which results in a detector response spreading inferior to the Poissonian spreading, will be very hard to study. The same happens when the studied effect is hidden beneath another stronger one.

The effect of limited quantum efficiency on the detector response spreading illustrated in section 3.2 is a good example. As explained previously, the statistical behaviour attached to this 
effect can be described by a binomial distribution law. Since a binomial distribution has a spread $\sigma_{\mathrm{QE}}=\sqrt{\mathrm{n} \cdot \mathrm{QE} \cdot(1-\mathrm{QE})}$, with $\mathrm{n}$ the number of photons, and $\mathrm{QE}$ the quantum efficiency of the detector, it is very hard to identify this effect against the "natural" Poissonian fluctuations of the signal of standard deviation $\sigma_{\text {poisson }}=\sqrt{\mathrm{n}}>\sigma_{\mathrm{QE}}$.

So HORUS makes it easy to understand and illustrate some basic behaviours of detectors which remain in most cases invisible to the user because of their relative low importance with respect to other effects. We believe this can be very valuable for new detector physicists.

\section{Summary}

HORUS is a detector simulation program, enabling a complete simulation of the detection process. HORUS computes the expected modifications of an input image, taking into account the detector geometry and dead areas, the sensor, and ASIC and ADC imperfections.

The program has a modular and pluggable structure, making it easy to deactivate or modify each of the processes implemented. Program runtime options can also be used to control each of the processes.

The specific implementation of HORUS for the XFEL-AGIPD was described and some examples of studies on the AGIPD characteristics were given. HORUS is now used to study in details the impact of the AGIPD on certain scientific experiments to be carried out at the future European XFEL facility, and to support the AGIPD development program.

The program is an excellent tool for guiding the decision process between conflicting scientific requirements and technological limits. Finally we would like to point out the potential educational value of HORUS for new detector physicists.

HORUS is available on requests, by contacting the authors.

\section{Acknowledgments}

The authors would like to thank the European XFEL for the funding of these developments, as well as Franz Pfeiffer and Gerhard Gruebel for fruitful discussions on the XFEL detector requirements.

\section{References}

[1] M. Altarelli et al., European X-ray free electron laser technical design report, The European XFEL, (2007).

[2] M. Cornacchia et al., Future possibilities of the Linac Coherent Light Source, J. Synchrotron Radiat. 11 (2004) 227.

[3] T. Shintake et al., Stable operation of a self-amplified spontaneous-emission free-electron laser in the extremely ultraviolet region, Phys. Rev. Spec. Top.-Ac. 12 (2009) 070701.

[4] European XFEL website, http://www.xfel.eu/.

[5] A.Korn et al., Investigation of charge carrier transport and charge sharing in X-ray semiconductor pixel detectors such as Medipix2, Nucl. Instrum. Meth. A 576 (2007) 239.

[6] P. Wiacek et al., Comprehensive simulation of the response of a silicon strip detector for position-sensitive measurements of X-rays, Nucl. Instrum. Meth. 551 (2005) 66. 
[7] H.-E Nilsson et al., Simulation of photon and charge transport in X-ray imaging semiconductor sensors, Nucl. Instrum. Meth. A 487 (2002) 151.

[8] G. Potdevin, U. Trunk and H. Graafsma, Performance simulation of a detector for 4th generation photon sources: the agipd, Nucl. Instrum. Meth. A 607 (2009) 51.

[9] See http://www.ittvis.com.

[10] S. Agostinelli, Geant4 a simulation toolkit, Nucl. Instrum. Meth. A 506 (2003) 250.

[11] F. Salvat et al., PENELOPE-2008: a code system for Monte Carlo simulation of electron and photon transport, in the Workshop Proceedings, June 30-July 3, Barcelona, Spain (2008), Nuclear Energy Agency No. 6416 (2009).

[12] G. Battistoni et al., The FLUKA code: description and benchmarking, in the proceedings of the Hadronic Shower Simulation Workshop, September 6-8, Batavia, U.S.A. (2006), AIP Conf. Proc. 896 (2007) 31.

[13] G.F. Knoll, Radiation detection and measurement, Willey \& Sons, U.S.A. (2000).

[14] H. Spieler, Semiconductor detector systems, Oxford Science Publications, Oxford U.K. (2008).

[15] J.Becker et al., Plasma effects in silicon detectors for the European XFEL and their impact on sensor performance, in the proceedings of New Developments in Radiation Detectors, $11^{\text {th }}$ European Symposium on Semiconductor Detectors, June 7-11, Wildbad Kreuth, Germany (200), submitted to Nucl. Instum. Meth.

[16] R.F. Fowler et al., Computational modelling of semiconducting X-ray detectors, Nucl. Instrum. Meth. A 477 (2002) 226. 